Biospotlight

\section{LIFE ON THE MEMBRANE}

Biologists interested in studying when, where, and how proteins interact in cells have a number of experimental options. There is the two-hybrid approach, the bimolecular fluorescence complementation assay (BiFC), fluorescence resonance energy transfer (FRET), the proximity ligation assay (PLA), or co-immunoprecipitation, to name a few. Each technique has specific benefits and drawbacks, especially when it comes to exploring membrane proteins where interactions can become unstable and difficult to detect during membrane extraction. In this issue, Joachim Denner and his colleagues from Berlin, Germany suggest a combination of methods to better monitor those membrane protein interactions that cause problems for some assays. When the researchers combined FRET and PLA, they found that their PLA data not only strongly correlated with FRET values, but also provided an additional readout with high spatial resolution. Denner and his team used their correlative FRET-PLA technique to explore the interactions between CD63 and a transmembrane protein, gp41 from HIV-1, demonstrating the potential of this new approach to examine membrane protein biology.

\section{See "Investigation of membrane protein-protein interactions using correlative FRET-PLA" on page 188.}

\section{CLOSING THE LID ON CONTAMINATION}

PCR generates millions upon millions of copies of the same template in a single-tube reaction. This can be a blessing but also a curse, since those millions of templates can lead to contamination of lab equipment if you're not careful. This is especially worrisome in forensics labs or labs working with ancient DNA, where the amounts of starting material are usually very small and even the slightest contamination can create large problems. So wouldn't it be great to not have to open a tube after amplification and still be able to visualize the results on an agarose gel? Nils Adey and his colleagues describe a clever engineering solution that actually makes this possible. Their Gel Contamination Control System works by puncturing the bottom of PCR tubes with a pin which is sealed, along with the tube, in a protective gel casing to avoid exposure to the surrounding environment. After the puncture, pressure within the tube forces the liquid through a channel in the pin leading to a well on an agarose gel where the PCR reaction can be visualized. After analysis, the entire system can be discarded, again avoiding any possible contamination. Although a prototype, the low cost and ability to avoid contamination make this system worth looking at for any lab concerned with amplification contamination.

\section{See "A gel electrophoresis loading system to prevent laboratory} contamination by amplification products" on page 199.

Selected and written by Nathan S. Blow, Ph.D. 四

BioTechniques 57:171 (October 2014) doi 10.2144/000114212

\section{HIGH-YIELD MASS SPECTROMETRY-BASED DETECTION OF BIOTINYLATED PROTEINS}

A popular way to analyze subproteomes is to biotinylate a spatially or temporally distinct population of proteins. Tagged proteins are affinity bound to beads, contaminants are washed away, and the remaining proteins are either digested in place or after elution. However, many untagged proteins are co-purified, because they interact nonspecifically with the beads or with the bead-bound proteins. Filtering out these contaminating "hits" is time-consuming, so Schiapparelli et al. developed direct detection of biotin-containing tags (DiDBiT) to solve this problem. The authors showed that digesting the sample before affinity purification dramatically increased the ratio of labeled to unlabeled peptides that were detected by mass spectrometry. In cells bulk labeled with biotin, DiDBiT identified 10 times more biotinylated proteins than the traditional method. When newly synthesized proteins were biotinylated (a much lower-abundance target), the best on-bead digestion approach detected only $5 \%$ of the tagged proteins found using DiDBiT. The new technique even enables detection of newly synthesized, biotin-tagged proteins in vivo.

\section{L.M. Schiapparelli et al. Direct detection of biotinylated proteins by mass spectrometry. J Proteome Res. 13:3966-78.}

\section{THIAZOLE ORANGE-BINDING APTAMER FOR FLUORESCENTLY TAGGING RNA}

The MS2 coat protein-GFP fusion is a well-established tool for fluorescently labeling RNA via an embedded MS2 hairpin, but it is limited by no fluorescence enhancement upon fluorophore-RNA binding. The Spinach aptamer and its fluorescent binding partner solve that problem, but at the cost of a less impressive dissociation constant. Now, Dolgosheina et al. offer "RNA Mango," which binds thiazole orange (TO1) biotin with a $\mathrm{K}_{d}$ of $3 \mathrm{nM}$ and a 1100-fold fluorescence enhancement. The authors show that the RNA Mango-TO1-biotin complex is sufficiently bright for single-molecule imaging in vitro. In vivo, co-injection of RNA Mango and TO1-biotin in C. elegans gives strong, stable fluorescence. The aptamer works within the context of a native RNA transcript, as shown by capture of overexpressed 6S RNA Mango from bacterial cell lysate by TO1-biotin-loaded streptavidin beads. Since both bacterial and metazoan cells are permeable to TO1-biotin, RNA Mango holds promise as a new RNA tracking tool.

E.V. Dolgosheina et al. RNA Mango aptamer-fluorophore: a bright, high-affinity complex for RNA labeling and tracking. ACS Chem Biol. [Epub ahead of print, August 21, 2014; doi:10.1021/cb500499x]

Selected and written by Nijsje Dorman, Ph.D. [ᄄ⿴囗十心

BioTechniques 57:171 (October 2014) doi 10.2144/000114212 